\title{
Thermal Diffusion and Mass Transfer Effects on MHD Flow of a Dusty Gas through Porous Medium
}

\author{
Rajesh Kumar, ${ }^{1}$ Devendra Kumar, ${ }^{2}$ and R. K. Shrivastav ${ }^{3}$ \\ ${ }^{1}$ Department of Mathematics, Eshan College of Engineering, Farah Mathura, India \\ ${ }^{2}$ Sachdeva Institute of Technology, Mathura, India \\ ${ }^{3}$ Department of Mathematics, Agra College, Agra, India \\ Correspondence should be addressed to Rajesh Kumar, 79rajesh.ece@gmail.com
}

Received 14 September 2011; Accepted 27 October 2011

Academic Editor: X. Wen

Copyright (c) 2012 Rajesh Kumar et al. This is an open access article distributed under the Creative Commons Attribution License, which permits unrestricted use, distribution, and reproduction in any medium, provided the original work is properly cited.

The present problem is concerned with the thermal diffusion mass transfer effects on MHD free convective flow of dusty gas through a porous medium induced by the motion of a semiinfinite flat plate moving with velocity decreasing "exponentially with time". The effects of various parameters like magnetic parameter $M$ thermal diffusion effect as soret number $S_{1}$, permeability parameter $K_{1}$, Schimdt number $S_{c}$ are taken into account. The velocity profile, temperature field, and concentration of incompressible dusty gas and dust particles for several parameters are discussed numerically and explained graphically.

\section{Introduction}

The thermal diffusion (commonly known as soret effect) for instance, has been utilized for isotope separation, and in mixtures between gases with very light-molecular-weight $\left[\mathrm{H}_{2}, \mathrm{He}\right]$ and the medium-molecular-weight $\left[\mathrm{N}_{2}\right.$, air] the diffusion thermoeffect was found to be of a magnitude such that it cannot be neglected, Eckert and Darke [1]. In view of the importance of this diffusion thermoeffect, recently Basant Kumar and Singh [2] studied the free convection and mass transfer flow in an infinite vertical plate moving impulsively in its own plane, taking into account the soret effect.

The problems of fluid mechanics involving gas particles mixture arise in many processes of practical importance. One of the earliest problems is that of the heat and mass transfer in the mist-flow region of a boiler tube. The liquid rocket is another example, usually the oxidizer vaporizes much more rapidly that the fuel spray and combustion occurs. Heterogeneously around each droplet, the length of the combustion chamber and the stability of 
the flow of acoustic or shock waves are practical two-phase flow problems. The study of the flow of dusty gases, which has gained increased attention recently has wide applications in environmental sciences; one finds in the literature an amazing number of derivations of equations for the flow of a gas particle mixture. The equations have been developed by the several authors for various special problem. Under various assumptions a few derivations primarily for the gas particle mixture are listed here, Saffman [3], Marble [4], and Soo [5]. Using the formulation of Saffman [3] several authors have given exact solutions of various dusty gas problems, Michael and Norey [6], Rao [7] Verma and Mathur [8], Singh [9], Rukmangadachari [10], and Mitra [11] Studied the problem of circular cylinders under various condition. Gupta [12] considered the unsteady flow of a dusty gas in a channel whose crosssection is an annular sector regarding the plate problems. Liu [13], Michael and Miller [14], Liu [15], and Vimal [16] studied the problems of infinite flat plate under various conditions. Mitra [17] has studied the flow of a dusty gas induced by the motion of a semi-infinite flat plate moving with velocity decreasing exponentially with time. Singh [18] has studied MHD flow of a dusty gas through a porous medium induced by the motion or a semi-infinite flat plate moving with velocity decreasing exponentially with time. Singh and Gupta [19] have discussed MHD free convective flow of a dusty gas through a porous medium induced by the motion of a semi-infinite flat plate moving with velocity decreasing exponentially with time. Recently, Singh and Varshney have seen the mass transfer effects on study of Singh and Gupta. In the present section, we are considering the problem of Singh and Varshney [20] taking thermal diffusion into account under the same conditions taken by Singh and Vaershney.

\section{Mathematical Formulation of the Problem and Its Solution}

We assume the dusty gas to be confined in the space $y>0$ and the flow is produced by the motion of the semi-infinite flat plate moving with velocity $v e^{-\lambda^{2} t}$ in $x$ direction, $x$-axis taken along the plate and $y$-axis to be measured normal to it. Since the plate is semi-infinite, all the physical quantities will be functions of $y$ and $t$ only. According to Saffman [3] the equation of motion of the dusty gas and the dust particles along the $x$-axis are, respectively, given by

$$
\begin{aligned}
& \frac{\partial u}{\partial t}=v \frac{\partial^{2} y}{\partial y^{2}}+\frac{K_{0} N_{0}}{\rho}(v-u), \\
& \frac{\partial v}{\partial t}=\frac{K_{0}}{m}(u-v), \\
& \frac{\partial T}{\partial t}=\frac{K_{T}}{\rho C_{P}} \frac{\partial^{2} T}{\partial y^{2}}, \\
& \frac{\partial C}{\partial t}=D \frac{\partial^{2} C}{\partial y^{2}}+D_{T} \frac{\partial^{2} T}{\partial y^{2}},
\end{aligned}
$$

where $u$ and $v$ denote the velocity of gas and dust particles, respectively, $v$ is the kinemetic coefficient of viscosity of the gas, $K_{0}$ is the stokes resistance coefficient, $N_{0}$ is the number density of the dust particles which is taken to be constant, $\rho$ is the density of the gas, $m$ is the mass of 
dust particle, $K_{T}$ is the thermal conductivity, $C_{P}$ is the specific heat at the constant pressure, $D$ is the molecular diffusivity, and $D_{T}$ is the thermal diffusivity.

Applying the magnetic field, porous medium, free convection, mass transfer, and thermal diffusion along the $x$-axis the equation of motion (2.1) reduces to

$$
\frac{\partial u}{\partial t}=v \frac{\partial^{2} u}{\partial y^{2}}+\frac{K_{0} N_{0}}{\rho}(v-u)-\frac{\sigma B_{0}^{2} u}{\rho}-\frac{v}{K} u+g \beta \theta+g \beta^{\prime} \phi,
$$

where

$$
\theta=T-T \infty, \quad \phi=C-C \infty
$$

The boundary conditions are

$$
\begin{aligned}
& \theta=v e^{-\lambda^{2} t}, \quad \phi=v e^{-\lambda^{2} t}, \quad u=v e^{-\lambda^{2} t}, \\
& \text { At } y=0, \quad \theta=0, \quad \varnothing=0 \quad \text { as } y \longrightarrow \infty .
\end{aligned}
$$

Let the nondimensionals introduced be

$$
\begin{gathered}
y^{*}=\frac{y}{(\gamma t)^{1 / 2}}, \quad u^{*}=\frac{u}{v^{\prime}} \quad v^{*}=\frac{v}{v^{\prime}} \quad t^{*}=\frac{t}{\tau}, \\
\tau=\frac{m}{K_{0}}, \quad \theta^{*}=\frac{\theta}{v^{\prime}} \quad \phi^{*}=\frac{\phi}{v} .
\end{gathered}
$$

On applying nondimensionals the dimensionless forms of (2.5), (2.2), (2.3), and (2.4) are, respectively,

$$
\begin{gathered}
\frac{\partial u}{\partial t}=\frac{\partial^{2} u}{\partial y^{2}}+f(v-u)-M u-\frac{1}{K_{1}} u+\beta_{1} \theta+\beta_{2} \phi, \\
\frac{\partial v}{\partial t}=(u-v), \\
\frac{\partial \theta}{\partial t}=\frac{1}{P_{r}} \frac{\partial^{2} \theta}{\partial y^{2}} \\
\frac{\partial \phi}{\partial t}=\frac{1}{S_{c}} \frac{\partial^{2} \phi}{\partial y^{2}}+\frac{1}{S_{1}} \frac{\partial^{2} \theta}{\partial y^{2}}
\end{gathered}
$$

where $f$ is the mass of concentration of dust particles, $M$ is the magnetic parameter, $\beta_{1}$ volumetric expansion parameter, $\beta_{2}$ is the mass expansion parameter, $S_{c}$ is the Schmidt number, $P_{r}$ 
is the prandtl number, $K_{1}$ is the permeability parameter, and $S_{1}$ is the thermal diffusion parameter as soret number

$$
\begin{aligned}
& f=\frac{m N_{0}}{\rho}, \quad M=\frac{m \sigma B_{0}^{2}}{K_{0} \rho}, \quad \beta_{1}=g \beta \tau, \quad \beta_{2}=g \beta^{\prime} \tau, \\
& S_{c}=\frac{v}{D}, \quad P_{r}=\frac{\rho v C_{p}}{K \tau}, \quad \frac{1}{K_{1}}=\frac{v T}{K}, \quad S_{1}=\frac{v}{D_{T}} .
\end{aligned}
$$

The boundary condition (2.7) is reduced to

$$
\begin{gathered}
\theta=e^{-\lambda^{2} t}, \quad \phi=e^{-\lambda^{2} t}, \quad u=e^{-\lambda^{2} t} \text { at } y=0 \\
\theta \longrightarrow 0, \quad \phi \longrightarrow 0, \quad u \longrightarrow 0 \quad \text { as } y \longrightarrow \infty
\end{gathered}
$$

Let us choose the solutions of (2.9), (2.10), (2.11), and (2.12), respectively, as

$$
\begin{aligned}
& u=F(y) e^{-\lambda^{2} t}, \\
& v=G(y) e^{-\lambda^{2} t}, \\
& \theta=H(y) e^{-\lambda^{2} t} \\
& \phi=I(y) e^{-\lambda^{2} t}
\end{aligned}
$$

Using the solution the boundary condition (2.14),

$$
\begin{gathered}
H=1, \quad I=1, \quad F=1 \quad \text { at } y=0, \\
H \longrightarrow 0, \quad I \longrightarrow 0, \quad F \longrightarrow 0 \quad \text { at } y \longrightarrow \infty .
\end{gathered}
$$

By virtue of (2.15), (2.16), (2.17), and (2.18), (2.9), (2.10), (2.11), and (2.12) are, respectively, reduced to

$$
\begin{gathered}
\frac{d^{2} F}{d y^{2}}+f \cdot G+F\left(\lambda^{2}-f-M-\frac{1}{K_{1}}\right)=-\beta_{1} H-\beta_{2} I \\
G\left(1-\lambda^{2}\right)=F \\
\frac{d^{2} H}{d y^{2}}+\lambda^{2} H P_{r}=0 \\
\frac{d^{2} I}{d y^{2}}+\frac{d^{2} H}{d y^{2}}+\lambda^{2} I S_{1} S_{c}=0 .
\end{gathered}
$$


Eliminating $G$ from (2.20) and (2.21) we get

$$
\begin{gathered}
\frac{d^{2} F}{d y^{2}}+\frac{f F}{1-\lambda^{2}}+F\left(\lambda^{2}-f-M-\frac{1}{K}\right)=-\beta_{1} H-\beta_{2} I \\
\frac{d^{2} F}{d y^{2}}+n^{2} F=-\beta_{1} H-\beta_{2} I
\end{gathered}
$$

where $n=\left[\left(\lambda^{4}-\lambda^{2}\left(1+f+M+K_{1}^{-1}\right)+M+K_{1}^{-1}\right) /\left(\lambda^{2}-1\right)\right]^{1 / 2}$.

From (2.22), we get

$$
H=e^{-i s y}
$$

where $s=\lambda \sqrt{P_{r}}$.

From (2.23)

$$
I=\left(1-\frac{s^{2}}{m^{2}-s^{2}}\right) e^{-i m y}+\frac{s^{2}}{m^{2}-s^{2}} e^{-i s_{1} y}
$$

where $s_{1}=\lambda \sqrt{S_{c}}$.

By the boundary condition (2.19) the solution of (2.25) is obtained as

$$
F=e^{-i n y}+\frac{\beta_{1}}{n^{2}-s^{2}}\left(e^{-i n y}-e^{-i s y}\right)+\frac{\beta_{2}}{n^{2}-s_{1}^{2}}\left(e^{-i n y}-e^{-i s_{1} y}\right) .
$$

Then from (2.15) we get the velocity of dusty gas as

$$
u=\left[e^{-i n y}+\frac{\beta_{1}}{n^{2}-s^{2}}\left(e^{-i n y}-e^{-i s y}\right)+\frac{\beta_{2}}{n^{2}-s_{1}^{2}}\left(e^{-i n y}-e^{-i s_{1} y}\right)\right] e^{-\lambda^{2} t} .
$$

Real part of $u$ is given by

$$
u=e^{-\lambda^{2} t} \cos n y+\frac{\beta_{1}}{n^{2}-s^{2}} e^{-\lambda^{2} t}(\cos n y-\cos s y)+\frac{\beta_{2}}{n^{2}-s_{1}^{2}} e^{-\lambda^{2} t}\left(\cos n y-\cos s_{1} y\right) .
$$

Using (2.16), (2.21), and (2.28) the real part of velocity of dust particle $v$ is obtained as

$$
v=\frac{1}{1-\lambda^{2}}\left[e^{-\lambda^{2} t} \cos n y+\frac{\beta_{1}}{n^{2}-s^{2}} e^{-\lambda^{2} t}(\cos n y-\cos s y)+\frac{\beta_{2}}{n^{2}-s_{1}^{2}} e^{-\lambda^{2} t}\left(\cos n y-\cos s_{1} y\right)\right] .
$$

Using (2.26) temperature distribution is given by

$$
\theta=e^{-i s y} e^{-\lambda^{2} t}
$$



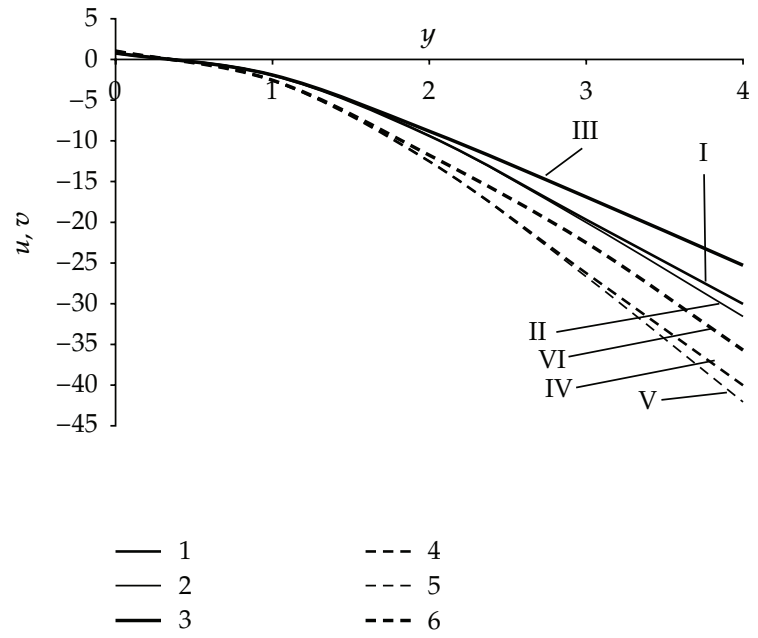

Figure 1: Velocity profile for $\lambda=.5, f=.2, P_{r}=.71, \beta_{1}=5, \beta_{2}=2, S_{1}=.38, S_{c}=.4$ at the different values of $M, K_{1}, S_{c}$, and $t$.

Real part of $\theta$

$$
\theta=e^{-\lambda^{2} t} \cos s y
$$

Using (2.27), concentration is given by

$$
\phi=\left[\left(1-\frac{s^{2}}{m^{2}-s^{2}}\right) e^{-i m y}+\frac{s^{2}}{m^{2}-s^{2}} e^{-i s_{1} y}\right] e^{-\lambda^{2} t}
$$

Real part of $\Phi$ is given by

$$
\phi=\left[\left(1-\frac{s^{2}}{m^{2}-s^{2}}\right) \cos m y+\frac{s^{2}}{m^{2}-s^{2}} \cos s_{1} y\right] e^{-\lambda^{2} t} .
$$

\section{Results and Discussion}

From the solid and dotted graphs of Figure 1 it is clear that velocity for dusty gas decrease with the increasing values of $y$ and increases with the increasing values of $t$. And for the increasing values of $\lambda$ keeping $y, t, M, S_{1}$ constant the velocity of dusty gas increases as well as the velocity of dust particles decreases. Increasing values of $t$ increases the velocity of dusty gas as well as the velocity of dust particles. From the solid and dotted graphs of Figure 2 it is noted that the temperature profile $\theta$ decreases and concentration profile $\phi$ increases when time $t$ is increases. From the solid and dotted graphs of Figure 3 it is noted that the temperature profile decreases and the concentration profile $\phi$ increases as thermal diffusion $S_{1}$ parameter as soret number increases at $\lambda=.5, f=.2, P_{r}=.71, \beta_{1}=5, \beta_{2}=2$. 

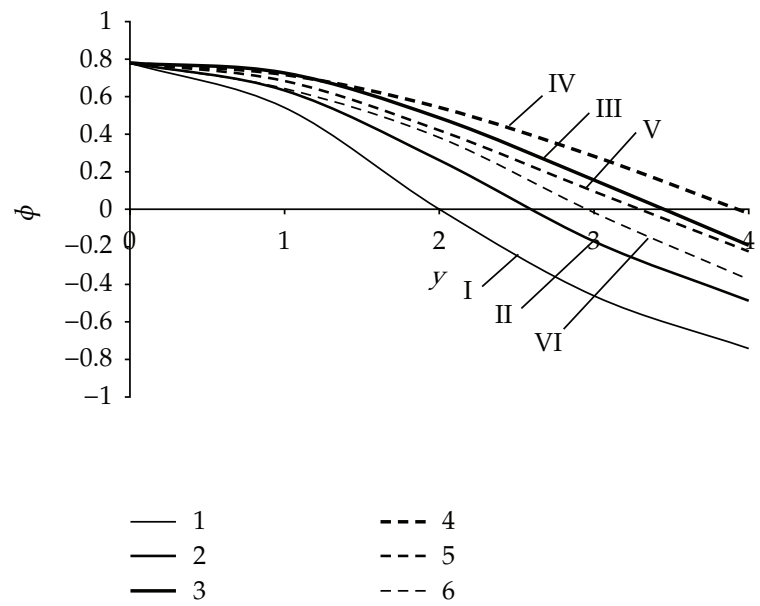

Figure 2: Concentration profile $\lambda=.5$ and $S_{c}=.6$ and the different values of $S_{1}$ and $t$.

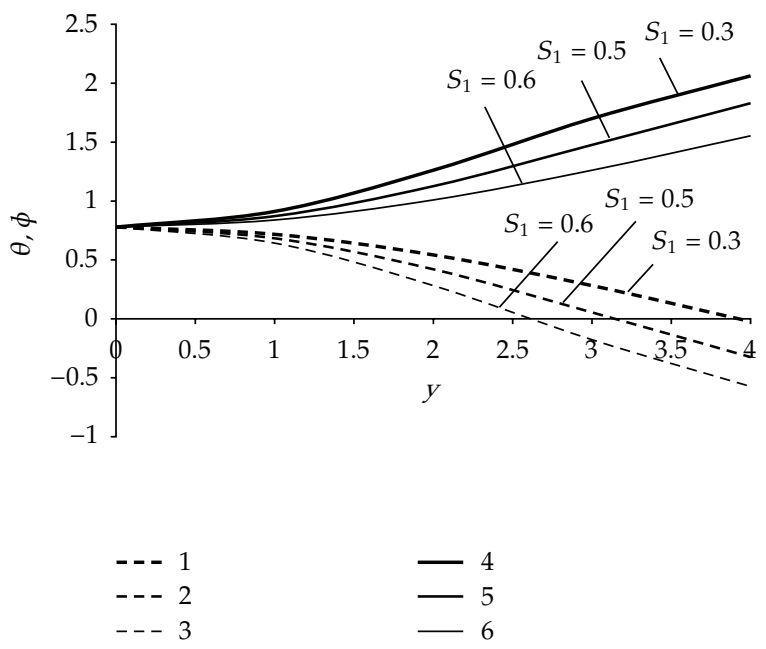

Figure 3: Concentration and temperature profile $\lambda=.5, S_{c}=.6$ and different values of $S_{1}$ and $t$ are $S_{1}=$ $.3, .4,5, .6$ (see Table 3 ).

The velocity profile for dusty gas is tabulated in Table 1 and plotted in Figure 1 having solid graphs 1 to 3 for $t=1$ and dotted graphs 4 to 6 at $t=3$ and $\lambda=.5, f=.2, P_{r}=.71, \beta_{1}=5$, $\beta_{2}=2$ and different values of $M, K_{1}, S_{c}$, and $t$ are as in Table 4 .

\section{Conclusion}

We conclude our study on thermal diffusion as well as mass transfer effect on MHD free convective flow of a dusty gas through a porous medium induced by the motion of a semi-infinite flat plate moving with velocity decreasing exponentially with time as follows. 
Table 1: Velocity profile of dusty fluid and dust particle at $\lambda=.5, f=.2, P_{r}=.71, \beta_{1}=5, \beta_{2}=2, S_{1}=.38$, and $S_{c}=.4$ at the different values of $M, K_{1}, S_{c}$, and $t$.

\begin{tabular}{lcccccc}
\hline$Y$ & Graph 1 & Graph 2 & Graph 3 & Graph 4 & Graph 5 & Graph 6 \\
\hline 0 & 0.7788 & 0.7788 & 0.7788 & 1.0384 & 1.0384 & 1.0384 \\
1 & -1.93407 & -1.90368 & -1.91017 & -2.57876 & -2.53823 & -2.5469 \\
2 & -9.39053 & -9.37132 & -8.81633 & -12.5207 & -12.4951 & -11.7551 \\
3 & -19.6764 & -20.0147 & -16.8816 & -26.2352 & -26.6863 & -22.5088 \\
4 & -30.0193 & -31.554 & -25.2733 & -40.0257 & -42.072 & -35.6977 \\
\hline
\end{tabular}

Table 2: The concentration profile is tabulated in Table 2 plotted in Figure 2 having graphs 1 to 3 at $\lambda=.5$ and different values of $S_{1}$ and $t$ are taken for velocity values of concentration at $\lambda=.5, S_{c}=.6$ and the different values of $S_{1}$ and $t$.

\begin{tabular}{ccccccc}
\hline$Y$ & Graph 1 & Graph 2 & Graph 3 & Graph 4 & Graph 5 & Graph 6 \\
\hline 0 & 0.7788 & 0.7788 & 0.7788 & 0.7788 & 0.7788 & 0.7788 \\
1 & 0.54152 & 0.63284 & 0.72612 & 0.71732 & 0.68346 & 0.64277 \\
2 & 0.00017 & 0.26157 & 0.5473 & 0.5426 & 0.42079 & 0.2822 \\
3 & -0.46131 & -0.17084 & 0.20561 & 0.2822 & 0.05509 & 0.17695 \\
4 & -0.74208 & -0.48685 & -0.29205 & -0.02274 & -0.3241 & 0.57428 \\
\hline
\end{tabular}

Table 3: As taken for velocity, values of concentration profile $\lambda=.5, S_{c}=.6$ and different values of $S_{1}$ and $t$ are $S_{1}=.3, .4, .5, .6$.

\begin{tabular}{ccccccc}
\hline $\mathrm{y}$ & Graph 1 & Graph 2 & Graph 3 & Graph 4 & Graph 5 & Graph 6 \\
\hline 0 & 0.7788 & 0.7788 & 0.7788 & 0.7788 & 0.7788 & 0.7788 \\
1 & 0.71732 & 0.68346 & 0.64277 & 0.83886 & 0.8716 & 0.91158 \\
2 & 0.5426 & 0.42079 & 0.2822 & 1.00911 & 1.12606 & 1.26137 \\
3 & 0.2822 & 0.05509 & -0.17695 & 1.26137 & 1.47629 & 1.69947 \\
4 & -0.02274 & -0.3241 & -0.57428 & 1.55363 & 1.83056 & 2.06203 \\
\hline
\end{tabular}

Table 4: Different values of $M, K_{1}, S_{c}$, and $t$ taken for various graphs.

\begin{tabular}{lcccccc}
\hline & Graph 1 & Graph 2 & Graph 3 & Graph 4 & Graph 5 & Graph 6 \\
\hline$M$ & 0.1 & 0.2 & 0.1 & 0.1 & 0.1 & 0.2 \\
$K_{1}$ & 10 & 10 & 20 & 10 & 10 & 10 \\
$S_{c}$ & 0.4 & 0.4 & 0.4 & 0.8 & 0.4 & 0.4 \\
$t$ & 1 & 1 & 1 & 1 & 3 & 3 \\
\hline
\end{tabular}

(1) Increasing the viscoelastic parameter $(\lambda)$ increases the velocity of dusty gas and decreases the velocity of dust particles as well as decreases the temperature profile and increases the concentration profile.

(2) Increasing values of $y$ decreases the velocity of dusty gas while it increases the velocity of dust particles.

(3) Increasing values of thermal diffusion parameters as soret number $\left(S_{1}\right)$ decreases the temperature profile $(\theta)$ and increases the concentration profile $\phi$. 


\section{Acknowledgment}

The authors are highly thankful to provide to valuable suggestions to improve the quality of research paper.

\section{References}

[1] R. M. Eckert and Darke, Analysis of Heat and Mass Transfer, McGraw Hill, New York, NY, USA, 1972.

[2] J. Basant Kumar and A. K. Singh, "Soret effects on free-convection and mass transfer flow in the stokes problem for a infinite vertical plate," Astrophysics and Space Science, vol. 173, no. 2, pp. 251-255, 1990.

[3] P. G. Saffman, "On the stability of laminar flow of a dusty gas," Journal of Fluid Mechanics, vol. 13, pp. 120-128, 1962.

[4] F. E. Marble, Annual Review of Fluid Mechanics, vol. 2, Annual Reviews, Inc., Palo Alto, Calif, USA, 1970.

[5] S. L. Soo, Fluid Dynamics of Multiphase Systems, Blaisdeel, Boston, Mass, USA, 1971.

[6] D. H. Michael and P. W. Norey, "The laminar flow of a dusty gas between two rotating cylinders," Quarterly Journal of Mechanics and Applied Mathematics, vol. 21, no. 3, pp. 375-388, 1968.

[7] S. S. Rao, "Unsteady flow of dust viscous liquid through circular cylinder," Def. Sci. Jour., vol. 19, p. $135,1969$.

[8] P. D. Verma and A. K. Mathur, "A numerical solution of the flow of a dusty viscous liquid through of a circular cylinder," Indian Journal of Pure and Applied Mathematics, vol. 4, p. 133, 1977.

[9] D. Sing, Indian Journal of Physics, vol. 47, p. 341, 1972.

[10] E. Rukmangadachari, "Dusty viscous flow through a cylinder rectangular cross-section under time dependent pressure gradient," Indian Journal of Pure and Applied Mathematics, vol. 9, no. 8, 1978.

[11] P. J. Mitra, Math. Phys. Sci., vol. 13, no. 4, 1979.

[12] S. C. Gupta, "Unsteady MHD flow in a circular pipe under transverse magnetic field," Indian Journal of Pure and Applied Mathematics, vol. 19, no. 6, 1979.

[13] J. T. C. Liu, "Flow of a dusty gas through a channel arbitrary time varying pressure gradient," Astronautica Actu., vol. 17, p. 851, 1972.

[14] D. H. Michael and D. A. Miller, "Oscillatory of a plate in a binary mixture of incompressible Newtonian fluid," Mathematika, vol. 13, p. 197, 1966.

[15] J. T. C. Liu, "Flow induced by an oscillating infinite flat plate in a dusty gas," Physics of Fluids, vol. 9, no. 9 , pp. 1716-1720, 1966.

[16] C. S. Vimal, Defence Science Journal, vol. 22, p. 231, 1972.

[17] P. Mitra, "Flow of non-Newtonian fluid," Acta Ciencia Indica, vol. 6, no. 3, p. 144, 1980.

[18] K. P. Singh, "Unsteady flow of a stratified viscous fluid through a porous medium between two parallel plates with variable magnetic induction," Acta Ciencia India, vol. 25, no. 4, 1999.

[19] P. Singh and C. B. Gupta, "Hall currents effects on MHD free convection flow with heat and mass transfer in the presence of radiation and heat source," Jour PAS, vol. 8, pp. 193-204, 2002.

[20] Singh and Varshney, Acta Ciencia Indica, vol. 32, no. 1, p. 301, 2005. 




Advances in

Operations Research

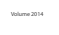

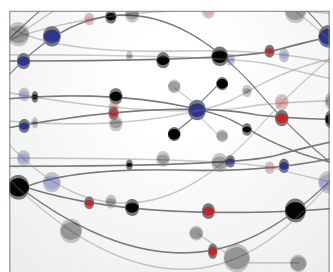

\section{The Scientific} World Journal
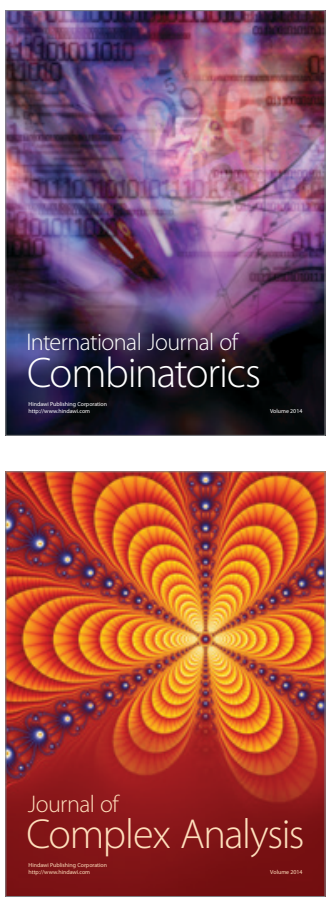

International Journal of

Mathematics and

Mathematical

Sciences
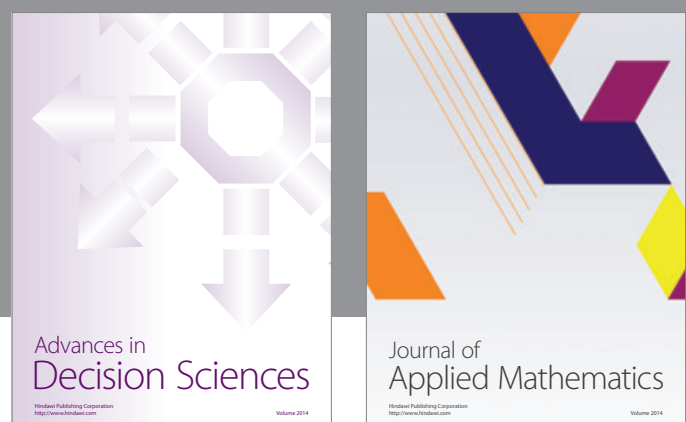

Journal of

Applied Mathematics
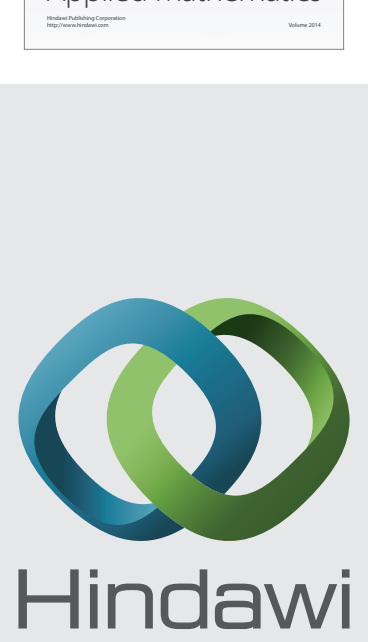

Submit your manuscripts at http://www.hindawi.com
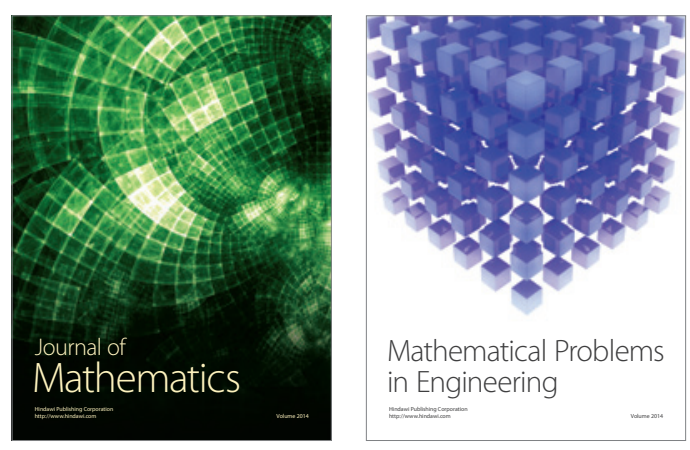

Mathematical Problems in Engineering
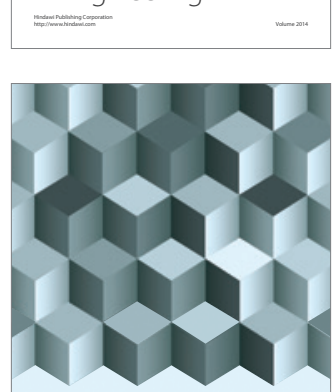

Journal of

Function Spaces
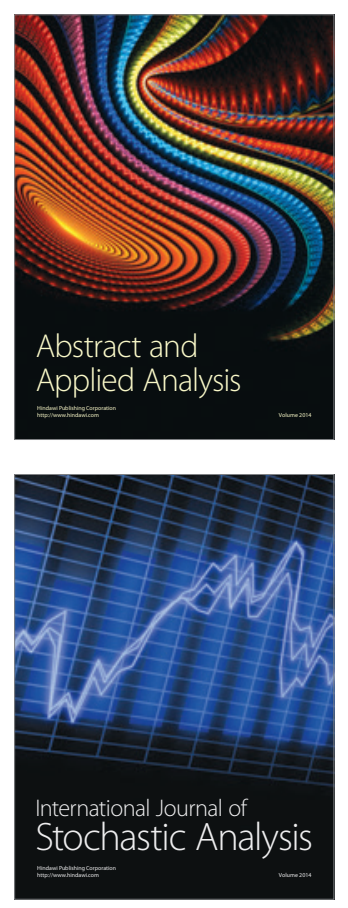

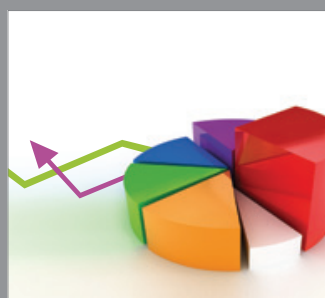

ournal of

Probability and Statistics

Promensencen
